\title{
CROMOBLASTOMICOSIS EN HUECO POPLÍTEO DERECHO, REPORTE DE CASO. PUEBLA, MÉXICO.
}

\author{
CHROMOBLASTOMYCOSIS IN RIGHT POPLITEAL FOSSA, CASE REPORT. \\ PUEBLA, MEXICO
}

\author{
Rodríguez Espinoza Mirsha Omar ${ }^{1}$, Aco Luna Jesús Alberto ${ }^{2}$, Norarizbeth Lara Flores ${ }^{3}$, Pris- \\ cila Rodríguez Espinosa ${ }^{4}$.
}

\section{RESUMEN}

La Cromoblastomicosis es una micosis subcutánea producida por hongos melanizados conocidos como dematiáceos, comúnmente secundario a un proceso traumático previo.Actualmente la incidencia de esta patología es desconocida. Se presenta el caso de un hombre de 64 años originario de la comunidad de Coyomeapan, Puebla, México, quien acude por falta de cicatrización de una úlcera en hueco poplíteo derecho generada por quemadura con candil de petróleo hace cuatro años. Refiriendo dolor con leve dificultad para la deambulación y sin evidencia de sangrado activo. Se realiza raspado de la lesión con presencia de células fumagoides posteriormente se aisló Cladophialophora carrionii en Agar Boreli. Se inició tratamiento con terbinafina para su recuperación. El conocimiento de este tipo de micosis aumenta la posibilidad de un diagnóstico temprano y certero para evitar complicaciones derivadas de la cronicidad o retraso del diagnóstico.

\section{ABSTRACT}

Chromoblastomycosis is a subcutaneous mycosis produced by pigment-producing fungi known as dematiaceous, commonly after a previous traumatic process. Currently the incidence of this pathology is unknown. We present the case of a 64-year-old man from the community of Coyomeapan, Puebla, Mexico, who came for lack of healing of an ulcer in the right popliteal socket generated by burning with an oil lamp four years ago. Referring pain with mild difficulty in ambulation and without evidence of active bleeding. Scraping of the lesion was performed with the presence of fumagoid cells. Cladophialophora carrionii was isolated on Boreli Agar. Treatment was started with terbinafina for recovery. The knowledge of this type of mycosis increases the possibility of an early and accurate diagnosis to avoid complications derived from the chronicity or delay of the diagnosis.

\section{INTRODUCCIÓN}

T a Cromoblastomicosis o Enfermedad de Fonseca Les una micosis subcutánea, crónica causada por hongos melanizados conocidos como dematiáceos ${ }^{1}$. Posee una distribución mundial frecuentemente encontrada en climas tropicales y subtropicales ${ }^{2}$ $y$ es ocasionada por hongos que generan color dematiáceos o feoides de los géneros Fonsecaea, Cladophialophora en su mayoría, aunque puede ser causada por otras especies ${ }^{3,5}$.

Padecimiento que se limita a piel y tejido subcutáneo, de desarrollo crónico y por lo regular se presenta en una sola forma clínica como nódulos verrugosos que se localizan más en miembros inferiores. Los hombres son los más afectados al igual que los campesinos. Cursa con lesiones verrugosas en forma de coliflor hasta producir lesiones incapacitantes ${ }^{1,5}$.
Existen diferentes factores que no favorecen al correcto diagnóstico de una entidad dermatológica, como el desconocimiento de la misma o la falta de reportes epidemiológicos.

\section{PRESENTACIÓN DEL CASO}

Hombre de 64 años, originario de Coyomepan, Puebla, de ocupación campesino, viudo. Acude a consulta rural del programa PROSAH (Proyecto de Salud Ambiental y Humana).

Se realiza interrogatorio indirecto mediante traductor para dialecto náhuatl, no presenta antecedentes de importancia.

El paciente acude a consulta por presentar lesión ulcerosa en hueco poplíteo derecho de tres años de
${ }^{1}$ Hospital General de Zona Sur, "Dr. Eduardo Vázquez Navarro". Servicios de Salud del Estado de Puebla.

${ }^{2}$ Médico Cirujano y Partero Agentes Biológicos, Facultad de Medicina, Benemérita Universidad Autónoma de Puebla

${ }^{3}$ Maestra en Ciencias Microbiología, Licenciada en Biomedicina, Médico Cirujano y Partero. Agentes Biológicos, Facultad de Medicina, Benemérita Universidad Autónoma de Puebla.

${ }^{4}$ Estudiante de la Licenciatura en Medicina Facultad de Medicina, Benemérita Universidad Autónoma de Puebla.

Correspondencia a:

Correspondencia a: Aco Luna Jesus Alberto

Correo: jealaclu@gmail.com Tel: +52 2222295500 Ext. 6058

Palabras clave: Cromoblastomicosis, úlcera, cicatriz. Keywords: Chromoblastomycosis, scars, ulcer.

Procedencia y arbitraje: no comisionado, sometido a arbitraje externo.

Recibido para publicación: 05 de marzo del 2019 Aceptado para publicación: 31 de Julio del 2019

Citar como: Rev Cient Cienc Med 2019; 22(1): 53-56 
evolución sobre cicatriz secundaria a quemadura de candil de petróleo hace cuatro años la cual no ha cicatrizado por completo.

A la exploración física se observa ulceración de márgenes bien definidos de poca profundidad (4 mm) de $2,3 \mathrm{~cm}$ por $1,2 \mathrm{~cm}$ de largo y ancho respectivamente, de fondo rojo sin secreción serosa o hemática(Figura 1). Se encuentra cicatriz retráctil perilesional, con descamación en bordes y aspecto blanquecino por aplicación de crema local. Escamas con puntilleo oscuro.

Presenta dolor a la movilización de la extremidad en intensidad 3 de 10, y dolor ardoroso moderado a la palpación 5 de 10 . No se encuentran adenomegalias regionales. Refiere aplicación de cicatrizante para caballos, ignorando el nombre del mismo, durante el último año sin mejoría.

Acorde a la ocupación y la zona geográfica se realiza toma de escamas perilesionales analizándolas microscópicamente con $\mathrm{NaOH}$ (hidróxido de sodio) al $15 \%$ encontrando Esclerotes de Medlar (Figura 2), correspondiendo con probable Cromoblastomicosis. El paciente fue tratado con Terbinafina para su pronta recuperación además de la aplicación de fomentos calientes para la disminución del tamaño de la lesión.

Se procedió a la realización del cultivo de las escamas en Agar de Borelli en el Laboratorio de Agentes Biológicos de la Facultad de Medicina BUAP identificándose a las 3 semanas Cladophialophora carrionii. (Figura 3 ).

\section{DISCUSIÓN}

En el grupo de las micosis subcutáneas se encuentran: el micetoma, la esporotricosis, la cromoblastomicosis, la lobomicosis y la rinosporidiosis ${ }^{4}$. La cromoblastomicosis es una entidad patológica causada por 5 diferentes géneros de hongos conocidos como dematiáceos, dos de estos géneros de hifomicetos melanizados son Cladophialophora y Fonsecaea, ambos pertenecen a la familia de los Herpotrichiellaceae en el orden de los Chaetothyriales, que son los agentes causales más comunes ${ }^{5}$ y ocasionalmente se han reportado casos ocasionados por especies de Phialophora, Exophiala, y Rhinocladiella, quienes también pertenecen a esta familia.

La melanina, es un importante factor de virulencia, interfiere con la activación del complemento reduciendo la susceptibilidad del pigmento fúngico a los antifúngicos ${ }^{1,6}$. Tras la inoculación por un trauma de la piel, infección local con crecimiento lento se desarrollan las lesiones ${ }^{6}$.

En una manifestación típica se forman las lesiones dérmicas como nódulos de coliflor. En el tejido infectado, característico de color oscuro, de paredes gruesas, las células muriformes es decir esclerotes (cuerpos de Medlar) ${ }^{7}$.

El prurito es relevante en el curso de esta enfermedad, ya que causa que el paciente contribuya a la inoculación del hongo en diferentes áreas, esto ayuda a que se ocasione una segunda infección, la cual es la complicación más frecuente de la cromoblastomicosis 8 .

Existen cinco formas clínicas de esta enfermedad: 1) Lesiones nodulares con superficie elevada y cubiertas por costras en forma de coliflor; 2) Lesiones tumorales extensas; 3 ) Lesiones verrucosas hiperqueratósicas irregulares extensas; 4) Placas superficiales escamosas, planas, rojizas; 5) Lesiones cicatrízales cutáneas con resguardo del centro.

Las formas más frecuentes son las lesiones hiperqueratósicas verrucosas y las nodulares $y$ pueden existir varios tipos a la vez ${ }^{10}$.

El diagnóstico citológico de la cromoblastomicosis requiere de la demostración de las células fumagoides o muriformes (Figura 2).

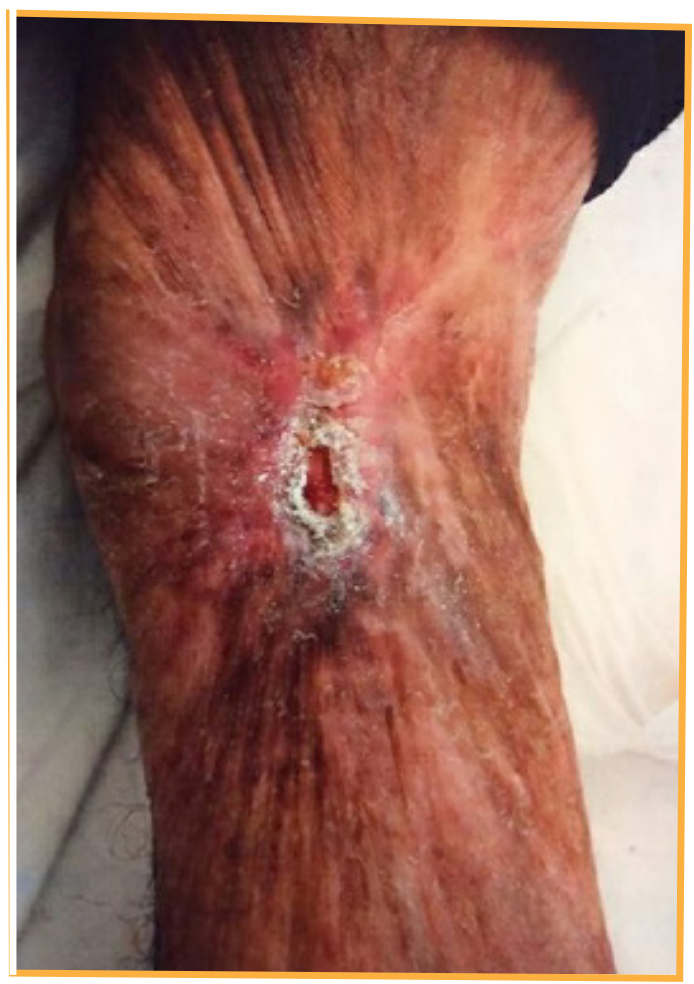

Figura 1: Lesión en hueco popliteo derecho. Cicatriz retráctil con úlcera secundaria 
en el material clínico incluyendo una Biopsia por Aspiración con Aguja Fina (BAAF) ${ }^{11}$ o en un examen directo con hidróxido de potasio $(\mathrm{KOH})$ al $10 \%$ y para corroborar el agente causal se utiliza medio de cultivo Borelli.

En el presente caso se detalla una cicatriz retráctil, ulcerosa haciéndose diagnóstico diferencial con una úlcera de Marjolin ya que en este caso se produjo una úlcera sobre una cicatriz previa en específico por quemadura $^{12}$.

Para su tratamiento se han realizado estudios acerca de la sensibilidad y resistencia de C. carrionii ante los antimicóticos, la cual es particularmente susceptible in vitro a los nuevos Azoles y a la Terbinafina, pero resistente a la Anfotericina B, Fluconazol, y Caspofungina ${ }^{6}$. La aplicación local de calor en la lesiones a temperaturas alrededor de $40-45^{\circ} \mathrm{C}$ permite la reducción en el tamaño de las lesiones y puede ser usado como tratamiento concomitante ${ }^{10}$. En casos donde la lesión es pequeña se puede usar la criocirugía o la cirugía para la extirpación de la lesión ${ }^{1,13}$.

$\mathrm{Su}$ pronóstico es bueno en casos superficiales, regular en resistencia a tratamiento y recidivas; y malo en variedades profundas hasta llegar a producir una incapacidad por el dolor o su localización ${ }^{1}$.

\section{Consideraciones Éticas:}

El presente reporte es de tipo descriptivo y no se realizaron tratamientos experimentales en el paciente. Se cuenta con consentimiento informado del paciente. Se ajusta a los lineamientos de la ley general de salud de México promulgada en 1986 y al código de Helsinki de 1975 y modificado en 1989, respecto a la confidencialidad de los participantes en el estudio, con registro del mismo en el comité de Investigación de la Facultad de Medicina de la Benemérita Universidad Autónoma de Puebla.

\section{Conflicto de Intereses.}

Los autores declaran no tener algún conflicto de intereses.

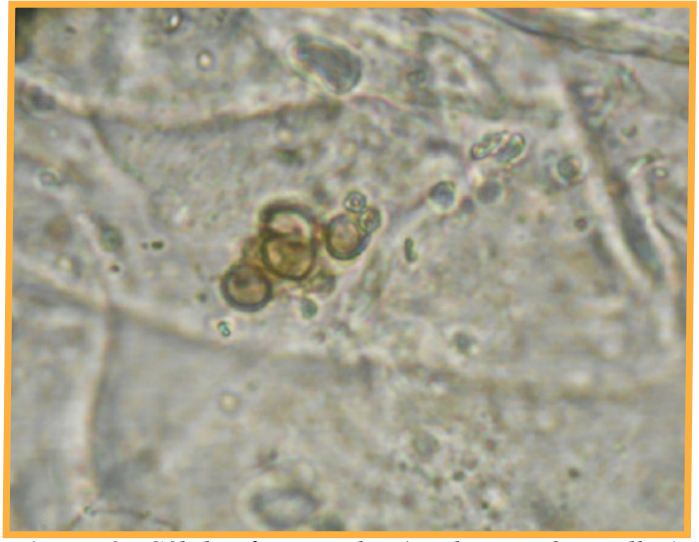

Figura 2: Células fumagoides (Esclerotes de Medlar) en observación directa con $\mathrm{NaOH}$.

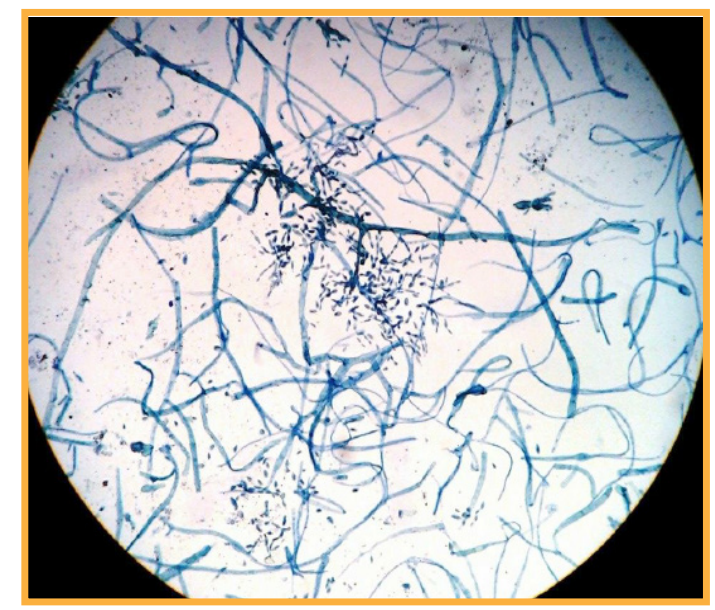

Figura 3: Cladophialophora carrionii 40X. Tinción con azul de metileno.

\section{Conclusiones.}

Al carecer de notificación epidemiológica, la incidencia real de cromoblastomicosis es incierta, por ello, cualquier caso confirmado microbiológicamente debe ser tomado como referente clínico y epidemiológico. El conocimiento de este tipo de micosis aumenta la posibilidad de un diagnóstico temprano y certero para evitar complicaciones derivadas de la cronicidad o retraso del diagnóstico. 


\section{REFERENCIAS}

1.-Bonifaz A. Cromoblastomicosis. En Bonifaz A. Micología Médica Básica 4a ed. México: McGraw-Hill; 2010. P. 231-246

2.- Torres-Guerrero E, Isa-Isa $\mathrm{R}$, Isa $\mathrm{M}$, Arenas $\mathrm{R}$ Chromoblastomycosis. Clinics in Dermat 2012;30(4): 403-408. doi:10.1016/j.clindermatol.2011.09.011 Disponible

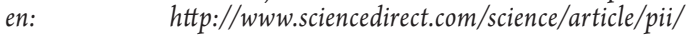
S0738081X11002902

3.- Romero NM, Arenas GR, Muñoz EVF, Atoche DCE, Mayorga RJ, Bonifaz A, Moraila MGF, et al. Cromoblastomicosis en México: Revisión de 603 casos en siete décadas. Dermatología CMQ 2014;12(2):87-93. Disponible en: http://new.medigraphic.com/cgi-bin/resumen.cgi ?IDREVISTA=323 - IDARTICULO $=54758 \leftrightarrow I D P U B L I C A C I$ $O N=5535$

4.- Bonifaz A, Vazquez GD, Perusquía OAM. Subcutaneous mycoses: Chromoblastomycosis, sporotrichosis and mycetoma.JDtsch Dermatol Ges.2010;8:619-27. doi:10.1111/ j.1610-0387.2010.07453.x Disponible en: http://onlinelibrary. wiley.com/doi/10.1111/j.1610-0387.2010.07453.x/abstract

5.- Deng S, de Hoog GS, Badali H, et al. In Vitro Antifungal Susceptibility of Cladophialophora carrionii, an Agent of Human Chromoblastomycosis. Antimicrobial Agents and Chemotherapy 2013;57(4):1974-1977. doi:10.1128/ AAC.02114-12. Disponible en: http://www.ncbi.nlm.nih.gov/ pmc/articles/PMC3623317/

6.- Jakopp B, Stamm B, Eyer D, Conen A. Hidden under a Cauliflower-Like Skin Tumor: Chromoblastomycosis. Case Reports in Infectious Diseases 2013;2013:450153. doi:10.1155/2013/450153. Disponible en: http://www.ncbi.nlm. nih.gov/pmc/articles/PMC3705846/

7.- Krzyściak PM, Pindycka-Piaszczyńska M, Piaszczyński M. Chromoblastomycosis. Advances in Dermatology and Allergology/Postpy Dermatologii i Alergologii
2014;31(5):310-321. doi:10.5114/pdia.2014.40949. Disponible en: http://www.ncbi.nlm.nih.gov/pmc/articles/PMC4221348/

8.- Bonifaz A, Carrasco-Gerard E, Saúl A. Chromoblastomycosis: clinical and mycologic experience of 51 cases. Mycoses 2001;44(1-2):-7. doi. 10.1046/j.1439. 0507.2001.00613.x. Disponible en: http://onlinelibrary.wiley. com/doi/10.1046/j.1439-0507.2001.00613.x/full

9.- López MR, Méndez TLJ. Chromoblastomycosis. Clinics in Dermatology 2007;25(2):188-194. doi:10.1016/j. clindermatol.2006.05.007. Disponible en: http://www. sciencedirect.com/science/article/pii/S0738081X06000691

10.-Bonifaz A. Micología Médica Básica. Cuarta edición. McGraw-Hill, México, D.F. 2012:231-246.

11.- Chavan SS, Reddy P. Cytological diagnosis of chromoblastomycosis. Journal of Cytology / Indian Academy of Cytologists 2013;30(4):276-277. doi:10.4103/09709371.126668. Disponible en: http://www.ncbi.nlm.nih.gov/pmc/ articles/PMC3945632/

12.- Morales Piñeiro Sergio, Morales Piñeiro Roberto, Morales Morera Tatiana. Úlcera de Marjolin. Presentación de un caso. Medisur [Internet].2016 Feb; 14( 1 ): 53-57.Disponible en: http://scielo.sld.cu/scielo.php?script=sci arttexte pid=S1727897X2016000100010wlng=es.

13.- Bada MM, Arenas GR, Vergara TL. Cromoblastomicosis en Veracruz. Un caso curado con extirpación quirúrgica. Med Int Mex 2012;28(2):192-195. Disponible en: $h t t p: / / w w w$. medigraphic.com/pdfs/medintmex/mim-2012/mim122m.pdf 